\title{
PERFIL DA FORMAÇÃO INICIAL E PERMANENTE DE TREINADORES DE TÊNIS DE ALTO RENDIMENTO DO BRASIL
}

\author{
Marcelo Bittencourt Neiva de Lima
}

Universidade do Estado de Santa Catarina, Florianopólis, Santa Catarina, Brasil

\author{
Alexandro Andrade \\ Universidade do Estado de Santa Catarina, Florianopólis, Santa Catarina, Brasil \\ Diego Itiberê Cunha Vasconcellos \\ Universidade do Estado de Santa Catarina, Florianopólis, Santa Catarina, Brasil \\ Mariana Bleyer de Faria \\ Universidade do Estado de Santa Catarina, Florianopólis, Santa Catarina, Brasil
}

\begin{abstract}
Resumo
O objetivo deste estudo foi descrever o perfil da formação inicial e permanente de treinadores de tênis de alto rendimento no Brasil. Participaram da amostra 24 treinadores do sexo masculino, com idades de 38,58 \pm 8,95 anos. Foi observado que a faixa etária dos treinadores de tênis é a adequada, apresentando muitos anos de prática de tênis e bom nível de jogo. A expertise adquirida pela prática parece dar suporte de intervenção para a grande maioria dos treinadores. Os treinadores de tênis fundamentam-se em aprendizagens informais e não-formais; participam de atividades de formação permanente, mas identificam limitações para participação, além de perceberem outras falhas organizacionais.
\end{abstract}

Palavras-chave: Formação de recursos humanos, tênis, desempenho atlético.

\section{Introdução}

Muitos estudos têm ratificado a importância da adequada formação profissional de treinadores para a obtenção de bons resultados no processo de treinamento e competição, além da importância da experiência que os treinadores devem ter, independente da formação acadêmica realizada (NORDMANN e SANDNER, 2009; JONES, 2006; SFARD, 1998; PIÉRON, 1993 e 1988; SIEDENTOP e ELDAR, 1989; BERLINER, 1986). A importância da formação de treinadores é reconhecida pelos próprios treinadores como fator fundamental para elevar a qualidade da prática profissional (VARGAS-TONSING, 2007) e pelos pesquisadores, que sugerem que trei-

Pensar a Prática, Goiânia, v. 17, n. 1, p. 01-294, jan./mar. 2014 1 
nadores melhores qualificados proporcionam formação esportiva mais apropriada e enriquecida qualitativa e quantitativamente aos seus atletas (KIRK e GORELY, 2000). O Departamento de Capacitação da CBT estimou que mil e quatrocentos professores haviam passado pelos cursos de capacitação. Deste total, 700 estavam filiados ao Departamento de Capacitação. Estimase que, no Brasil, existam cerca de sete mil professores de tênis (KIST, 2011). Entretanto, dados da CBT apontaram que somente 88 professores do Brasil graduaram-se no SNGP até o segundo mês de 2011 (CBT, 2010).

A percepção de que o Brasil carece de mais treinadores de tênis com formação adequada às necessidades do alto rendimento provém também de atletas, qual é o caso de Thomaz Bellucci. Em uma notícia vinculada no jornal Diário Catarinense, assim que rompeu com o último técnico, foi publicado o seguinte: “[...] O rompimento ocorre dois dias depois de Bellucci dizer que faltam técnicos competentes no Brasil [...] O tenista já foi comandado por três treinadores." (BELLUCCI, 2010, p. 8).

A preocupação com a formação de treinadores de outras modalidades esportivas é, entretanto, bastante estudada. O reconhecimento de que a qualidade da intervenção do treinador tem implicações importantes para a formação esportiva de atletas e para a obtenção dos melhores desempenhos esportivos, tem estimulado alguns países a desenvolver seus próprios programas de certificação ou educação formal de treinadores, como pressupõe os estudos de Nordmann e Sandner (2009), Lemyre et al. (2007), VargasTonsing (2007) e Wright et al. (2007), Côté (2006), Cushion et al. (2003), Cushion e Jones (2001), McCallister et al. (2000), Woodman (1993) e Gould et al. (1990), os quais apontam características comuns em sua estrutura e são oferecidos por uma gama de instituições, como universidades, associações profissionais, institutos, conselhos e federações esportivas, por exemplo (NORDMANN e SANDER, 2009; WRIGHT et al., 2007 e TRUDEL e GILBERT, 2006). No momento em que se reconhece e admite, pela crítica metodológica de investigação do ensino, que a conduta dos treinadores não é o único critério de eficácia pedagógica, o pensamento deles se mostra como um objeto de estudo relevante, com um potencial significativo, que necessita de novas técnicas e metodologias que nos permitam seguir avançando nos estudos sobre os processos de melhora e aperfeiçoamento dos treinadores em seus diferentes períodos de formação (RAMOS, 1999).

Para tanto, o objetivo deste estudo foi descrever o perfil do profissional e da formação inicial e permanente dos treinadores de tênis de alto rendimento no Brasil. 


\section{Método}

Este estudo caracteriza-se como descritivo e exploratório, utilizando a abordagem quantitativa para análise de dados (THOMAS e NELSON, 2002; CERVO e BERVIAN, 1983), e teve como amostra 24 treinadores de tênis competitivo do estado de Santa Catarina, todos do sexo masculino, com idades compreendidas entre 23 e 63 anos (38,58 \pm 8,95 anos). A amostra foi selecionada de forma não-probabilística intencional (THOMAS e NELSON, 2002). Somente participaram do estudo os treinadores que assinaram o Termo de Consentimento livre e Esclarecido, de acordo com o protocolo de aprovação do projeto de pesquisa pelo Comitê de Ética em Pesquisas com Seres Humanos (CEP) da Universidade do Estado de Santa Catarina - UDESC, registrado sob o n ${ }^{\circ}$ 147/2010.

Como instrumentos de pesquisa foram utilizados o Questionário de Caracterização Geral dos Treinadores de Tênis de Alto Rendimento e o "Questionário de Análise da Formação Inicial e Permanente dos Treinadores de Tênis de Alto Rendimento" (QUAFIPETAR), validado para a língua portuguesa do Brasil (LIMA, 2011).

O Questionário de Caracterização Geral dos Treinadores de Tênis de Alto Rendimento teve por objetivo apresentar características pessoais relevantes para o estudo, como idade, sexo, melhor ranking na ATP e WTA de um(a) tenista que tenha treinado, ano do melhor ranking do seu(sua) atleta, condições profissionais gerais, entre outros.

O QUAFIPETAR é um instrumento estruturado quantitativo que visa investigar a formação inicial e permanente de treinadores de tênis de alto rendimento. $\mathrm{O}$ questionário passou por processo de adaptação, testagem e validação para a língua portuguesa do Brasil pelos critérios de clareza, de validade de conteúdo aparente e fidedignidade (com valor alpha $(\alpha)$ de Cronbach de 0,84). É composto por 35 questões, sendo abertas, fechadas e, entre estas, com algumas questões utilizando uma escala Likert de 5 pontos ( 1 = absolutamente em desacordo, $2=$ em desacordo, $3=$ indiferente, $4=$ de acordo, e 5 = absolutamente de acordo).

O preenchimento do instrumento pelos treinadores aconteceu em um evento promovido pela Federação Catarinense de Tênis (FCT), em Itajaí (SC), sob a autorização prévia do presidente da federação. Todos os participantes foram informados sobre o estudo, sua relevância e de que forma poderiam contribuir para a pesquisa. Antes do preenchimento do instrumento foi esclarecido à todos que a participação era voluntária e não obrigatória.

Os dados foram tratados com estatística descritiva (valores médios, desvios padrão e valores mínimos e máximos) e inferencial. 


\section{Resultados e discussão}

O grupo que participou da validação de clareza do QUAFIPETAR foi composto por 24 treinadores de tênis competitivo do estado de Santa Catarina, todos do sexo masculino, com idades compreendidas entre 23 e 63 anos (38,58 $\pm 8,95$ anos), com experiência de 27,79 $\pm 11,10$ anos como tenistas e de $17 \pm 9,22$ anos como treinadores de tênis, com uma amplitude de experiência entre 2 e 40 anos entre a amostra. Declararam-se 88,2\% de etnia branca e $11,8 \%$ negra. O exame do gráfico de histograma revelou que as distribuições da idade são aproximadamente normais e que existe apenas um escore extremo (atípico) na variável (63 anos).

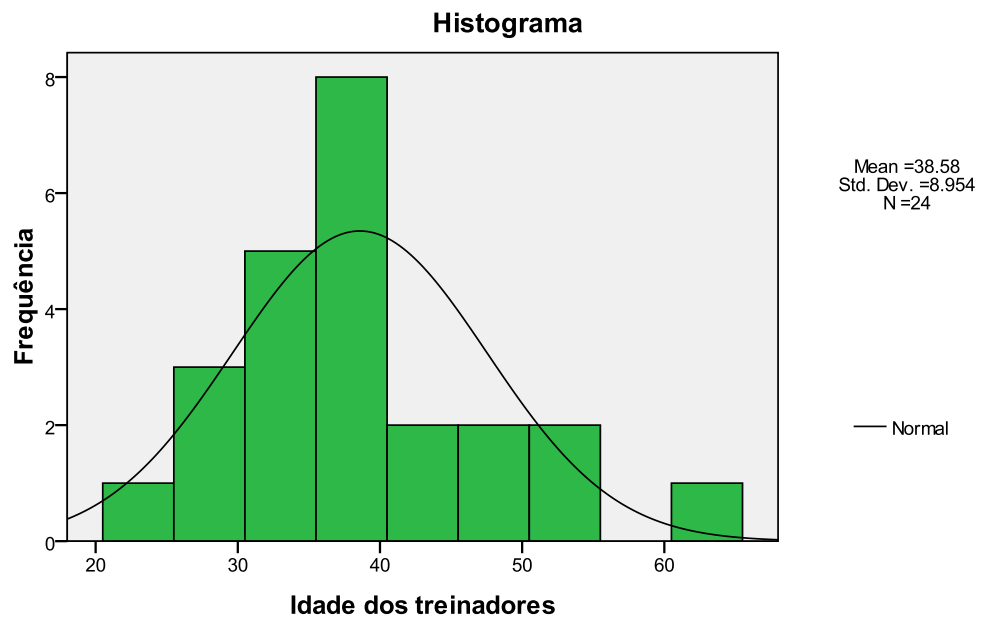

Gráfico 1. Histograma da distribuição da idade da amostra do estudo piloto

Os dados de idade dos treinadores encontrados neste estudo estão dentro de uma faixa indicada como ideal. Nos estudos de Resende (2009) e Ibañez et al (1997), que tratam sobre a idade mínima para ascender à carreira de treinador, os dados de referência são de 18 e 16 anos respectivamente. $\mathrm{O}$ consenso dos autores sobre a idade reside na necessidade de maturidade e domínio de diferentes áreas de intervenção. 
Tabela 1: Estatística descritiva das características pessoais dos treinadores do estudo.

\begin{tabular}{|c|c|c|c|c|c|}
\hline & $\mathbf{N}$ & $\overline{\bar{x}}$ & $\mathbf{D P}( \pm)$ & Mínimo & Máximo \\
\hline Idade (anos) & 24 & 38.58 & 8.95 & 23 & 63 \\
\hline $\begin{array}{l}\text { Melhor ranking da ATP de atleta profissional que } \\
\text { foi treinador }\end{array}$ & 9 & 621.78 & 512.20 & 26 & 1300 \\
\hline $\begin{array}{l}\text { Melhor ranking da WTA de atleta profissional } \\
\text { que foi treinador }\end{array}$ & 3 & 202.67 & 110.82 & 128 & 330 \\
\hline Ano em que o atleta atingiu o melhor ranking & 9 & 2001 & 8.37 & 1986 & 2010 \\
\hline Tempo de prática de tênis (anos) & 24 & 27.79 & 11.10 & 8 & 57 \\
\hline Tempo de prática (anos) como treinador de tênis & 23 & 17 & 9.22 & 2 & 40 \\
\hline
\end{tabular}

Onde: ${ }^{\bar{x}}$ representa a média e DP o desvio-padrão.

Dos 24 treinadores, onze treinaram atletas ranqueados entre $26^{\circ}$ e $1300^{\circ}$ na ATP ou WTA, oito afirmaram que nenhum dos seus atletas marcou pontos nos rankings profissionais e cinco deles não responderam sobre a questão. Dos treinadores, $83,3 \%$ trabalham somente com tênis, não exercendo nenhuma outra função profissional, enquanto que metade dos $16,7 \%$ restantes trabalha na área comercial e a outra metade em funções relacionadas com o tênis. A renda média é de até 3 salários mínimos (SM) para 8,3\% dos treinadores, 3 a 6 SM para $29,2 \%, 7$ a 10 SM para $41,7 \%$ e mais de 10 SM para $16,7 \%$ dos profissionais.

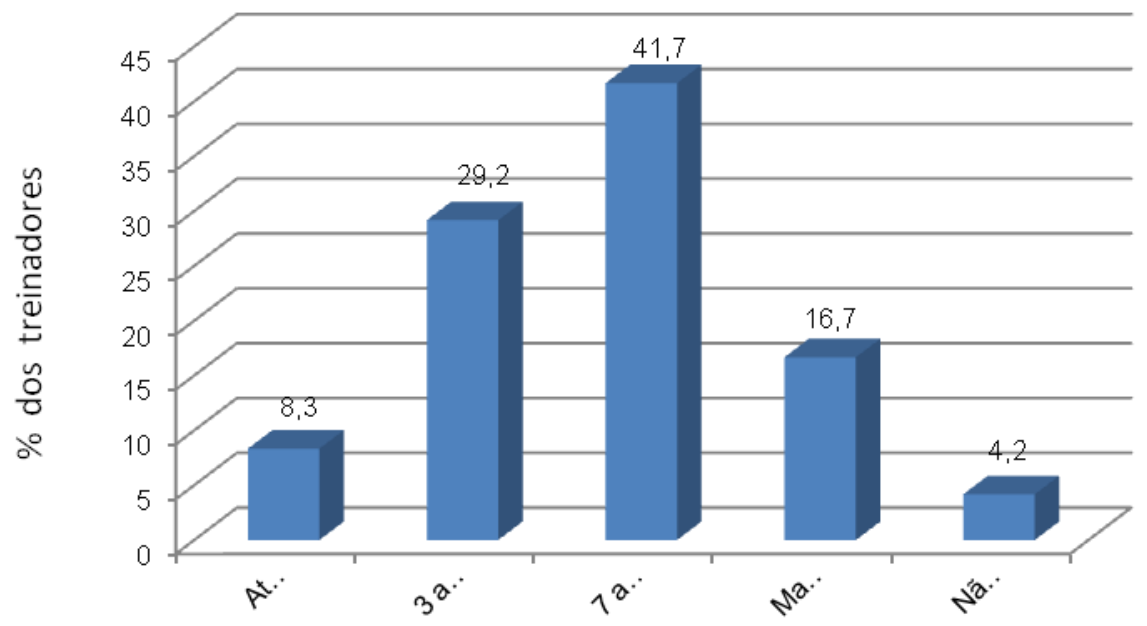

Gráfico 2. Distribuição de renda pessoal como treinador de tênis

Pensar a Prática, Goiânia, v. 17, n. 1, p. 01-294, jan./mar. 2014 
A média da remuneração mensal dos treinadores de tênis apresentouse consideravelmente maior que a média do rendimento mensal médio dos brasileiros ocupados que, segundo o IBGE (2011) é de pouco mais de um salário mínimo, com variações regionais entre $\mathrm{R} \$ 631,20$ no sudeste e de $\mathrm{R} \$ 314,70$ no nordeste.

Com relação ao nível de jogo do treinador enquanto tenista, observou-se que $87,5 \%$ deles jogou torneios no mais alto nível federativo do país e, ainda, $8,4 \%$ teve experiência na segunda e terceira classe, que representa um nível satisfatório de experiência ao professor. O perfil geral do nível de jogo dos treinadores aponta para uma ampla experiência com a prática dos fundamentos técnicos e táticos do jogo.

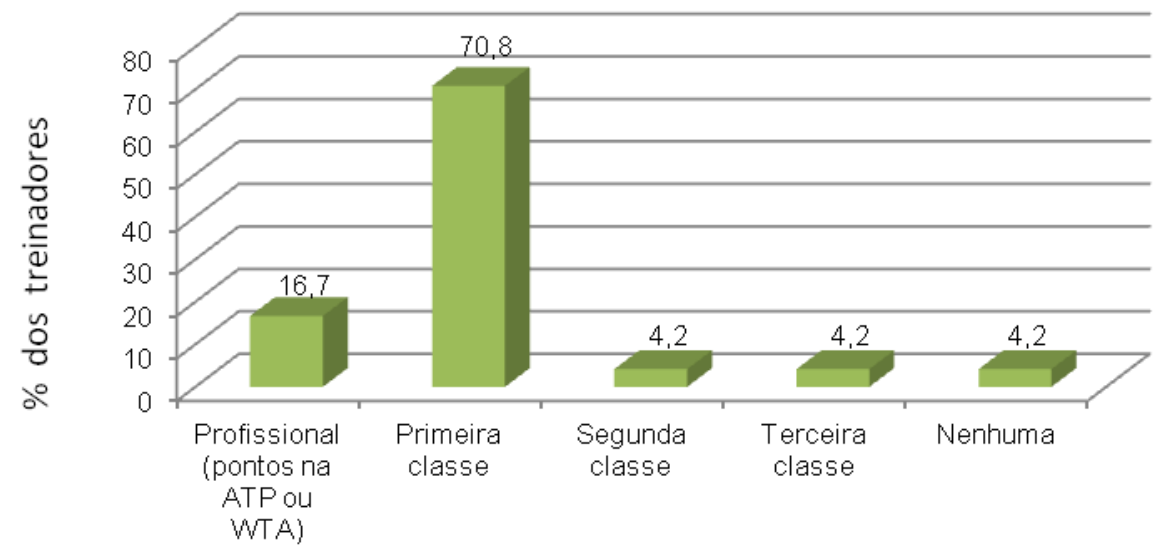

Nível de jogo

Gráfico 3. Distribuição por nível de jogo como jogador de tênis

Com referência à formação inicial formal (NELSON et al, 2006) dos treinadores, o perfil apresentado foi, nas áreas relacionadas com as Ciências da Atividade Física e do Esporte, de 22,7\% dos treinadores formados no curso de Educação Física. Destes, a maior graduação foi a especialização, com somente $4,5 \%$ dos profissionais. Os provisionados em tênis corresponderam a $77,3 \%$ dos treinadores.

Pensar a Prática, Goiânia, v. 17, n. 1, p. 01-294, jan./mar. 2014 
Tabela 2: Estatística descritiva das características de formação inicial dos treinadores relacionada com as Ciências da Atividade Física e Esportes.

\begin{tabular}{cccc}
\hline Graduação & $\mathbf{N}$ & $\%$ & $\begin{array}{c}\% \\
\text { acumulado }\end{array}$ \\
\hline Especialização & 1 & 4,5 & 4,5 \\
Licenciatura & 1 & 4,5 & 9,1 \\
Bacharelado & 2 & 9,1 & 18,2 \\
Licenciatura e Bacharelado & 1 & 4,5 & 22,7 \\
Ensino Médio & 8 & 36,4 & 59,1 \\
Nenhum dos anteriores & 9 & 40,9 & 100,0 \\
\hline
\end{tabular}

O baixo percentual de treinadores com graduação na área da Educação Física e Esportes preocupa, pois, segundo Cassidy et al (2009), boa parte da maestria profissional provém da aquisição de competências num período de formação educativa. Igualmente, Moreira (2005) apud Isidro (2009) indica que os treinadores que possuem uma formação superior em Educação Física apresentam uma noção mais aproximada das exigências de conhecimento teórico que não se adquire apenas da experiência da prática, ou nos cursos de aprendizagem formal iniciais.

Os dados do perfil de formação inicial também apontam que 52,2\% dos treinadores formaram-se no nível superior em áreas não relacionadas com as Ciências da Atividade Física e Esportes, bem como 47,8\% deles têm nível médio ou primário.

Tabela 3: Estatística descritiva das características de formação inicial dos treinadores não- relacionada com as Ciências da Atividade Física e Esportes.

\begin{tabular}{cccc}
\hline Graduação & $\mathbf{N}$ & $\boldsymbol{\%}$ & $\begin{array}{c}\% \\
\text { acumulado }\end{array}$ \\
\hline Especialização & 1 & 4,3 & 4,3 \\
Bacharelado & 3 & 13,0 & 17,4 \\
Licenciatura e Bacharelado & 8 & 34,8 & 52,2 \\
Ensino Médio & 8 & 34,8 & 87,0 \\
Nenhum dos anteriores & 3 & 13,0 & 100,0 \\
\hline
\end{tabular}

Confrontando os dados acima, observa-se que, tendo 47,8\% de treinadores com nível médio ou primário, 52,2\% com nível superior em áreas não relacionadas com as Ciências da Atividade Física e Esportes e 22,7\% com nível superior em Educação Física, então temos que, dentre os profissionais de nível superior não relacionados com as Ciências da Ativida 
de Física e Esportes, pouco menos da metade também é formado em Educação Física. Em um estudo sobre as condições de acesso à formação de treinadores em Portugal, Isidro (2009) manifestou dados em que os treinadores com outras formações que não em Educação Física consideraram mais importante a obtenção de uma formação acadêmica qualquer para acessarem à condição de treinadores, enquanto que os com formação superior em Educação Física enalteceram a importância de se obter formação específica em Educação Física para exercer a função de treinador.

Com relação à formação não-formal (NELSON et al, 2006) realizada pelos treinadores nos cursos da Confederação Brasileira de Tênis (CBT), observamos que apenas $29,2 \%$ dos treinadores da amostra não faziam parte do quadro de formação da CBT, pelo Sistema Nacional de Graduação Profissional (SNGP) da Confederação.

Tabela 4: Características de formação do tipo não formal (NELSON et al, 2006) dos treinadores pelo Sistema Nacional de Graduação Profissional (SNGP) da CBT

\begin{tabular}{cccc}
\hline Graduação & N & \% & $\begin{array}{c}\text { \% } \\
\text { acumulado }\end{array}$ \\
\hline Instrutor & 1 & 4,2 & 4,2 \\
Treinador & 6 & 25,0 & 29,2 \\
Treinador Nacional & 3 & 12,5 & 41,7 \\
Técnico Nacional & 3 & 12,5 & 54,2 \\
Técnico Master & 4 & 16,7 & 70,8 \\
Nenhum desses & 4 & 16,7 & 87,5 \\
Outro & 3 & 12,5 & 100,0 \\
\hline
\end{tabular}

Onde: Instrutor representa a menor graduação do sistema e Técnico Master representa a maior, respectivamente.

Entre os treinadores pesquisados, 70,8\% fazem parte do sistema, contrapondo os dados encontrados na CBT, onde somente 88 professores do Brasil graduaram-se no SNGP até o segundo mês de 2011 (CBT, 2010), em um universo estimado em cerca de sete mil professores de tênis (KIST, 2011).

Quando questionados sobre qual a titulação relacionada com as Ciências da Atividade Física e Esportes deveria ter qualquer professor das disciplinas específicas de tênis, em cursos de formação de treinadores, $65 \%$ manifestaram que deveriam ser, pelo menos, provisionados.

Pensar a Prática, Goiânia, v. 17, n. 1, p. 01-294, jan./mar. 2014 


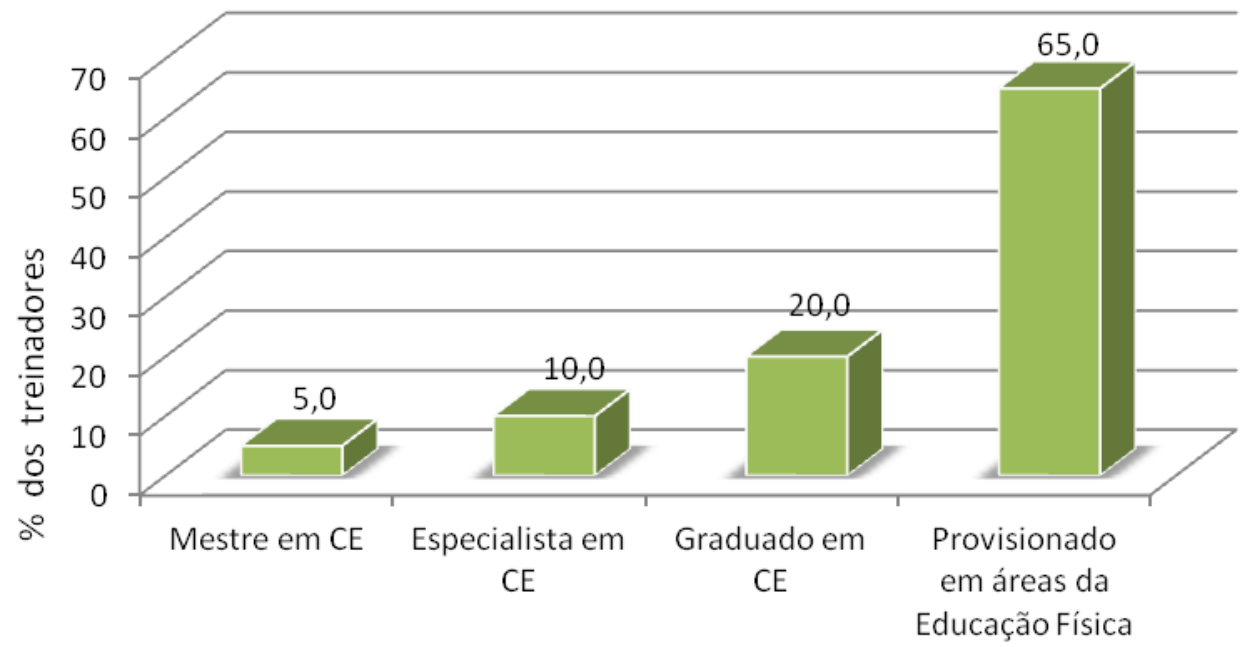

Titulação do professor responsável pelas disciplinas de tênis do curso de treinadores de tênis

Gráfico 4. Titulação necessária do professor responsável pelas disciplinas específicas de tênis do curso de treinadores de tênis, relacionada com as Ciências da Atividade Física e Esportes

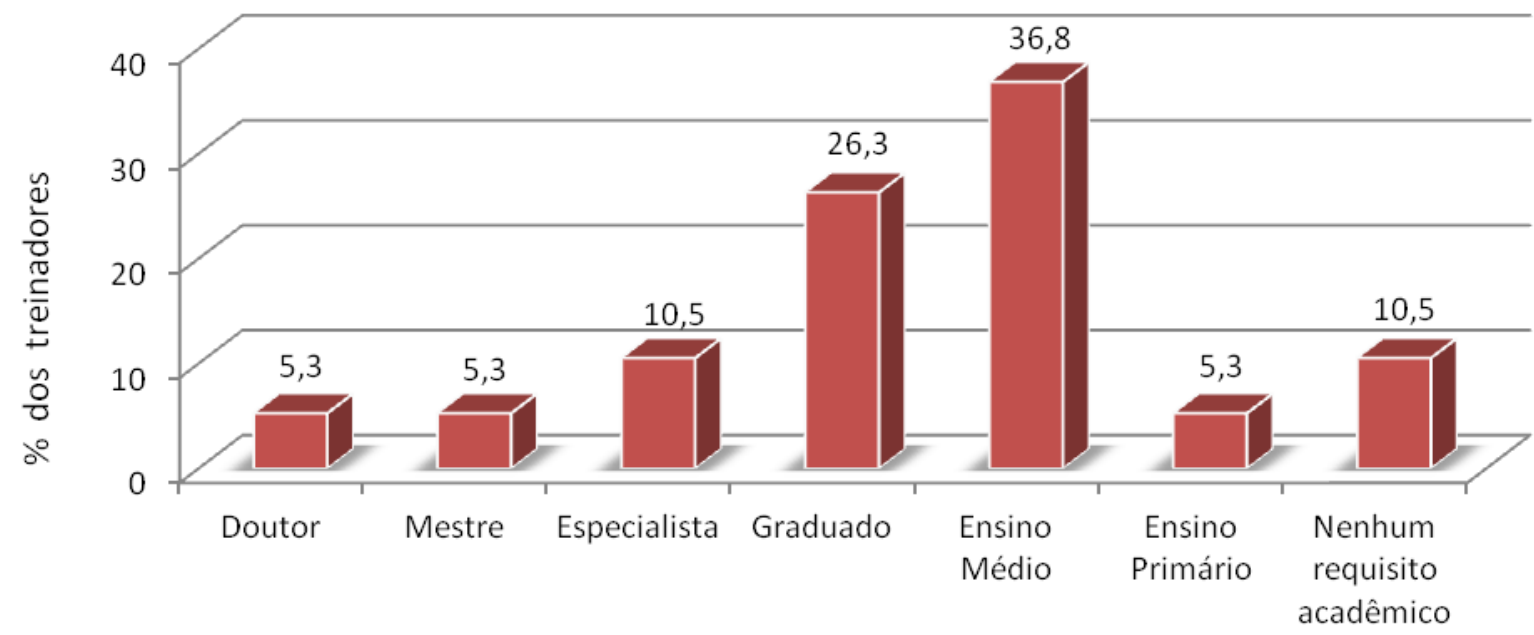

Titulação do professor responsável pelas disciplinas de tênis do curso de treinadores de tênis

Gráfico 5. Titulação necessária do professor responsável pelas disciplinas específicas de tênis do curso de treinadores de tênis, não-relacionada com as Ciências da Atividade Física e Esportes.

Pensar a Prática, Goiânia, v. 17, n. 1, p. 01-294, jan./mar. 2014 
Semelhantemente à opinião manifestada quanto ao professor responsável pelas disciplinas específicas de tênis do curso de treinadores de tênis, relacionada com as Ciências da Atividade Física e Esportes, os de área nãorelacionadas deveriam ser os de nível médio.

Quanto ao processo de formação permanente dos treinadores, em especial pelos caracterizados pela aprendizagem não-formal de Nelson et al (2006) (participação em cursos, congressos, palestras, entre outros), 68,4\% consideram entre boa e normal, sendo aperfeiçoável ou muito aperfeiçoável para $31,6 \%$ dos treinadores.

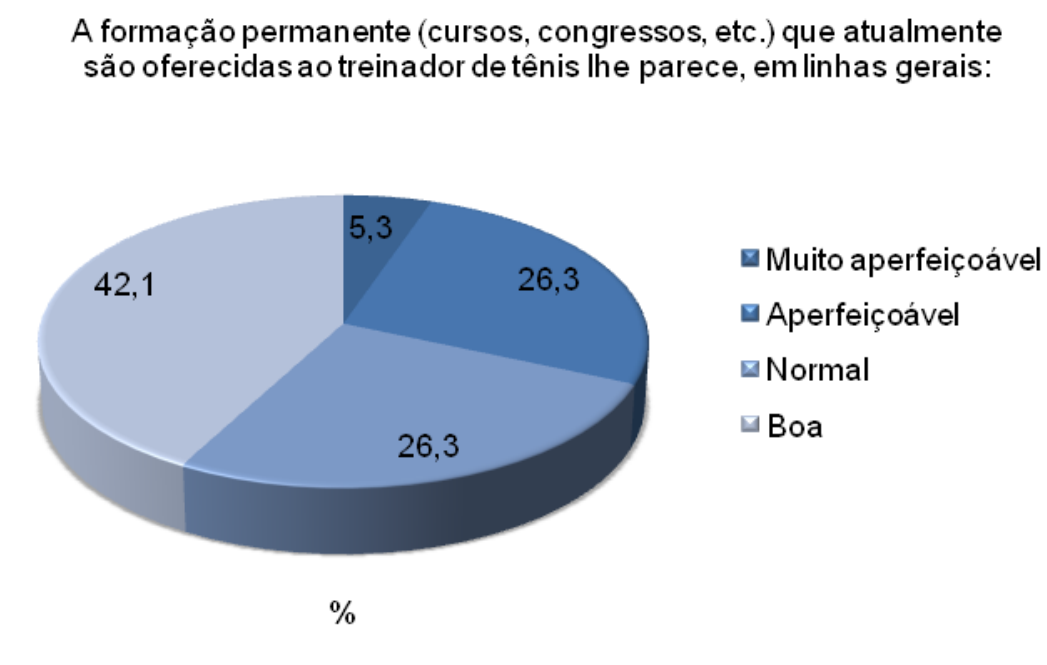

Gráfico 6. Opinião sobre a qualidade das atividades de formação permanente oferecidas aos treinadores de tênis 


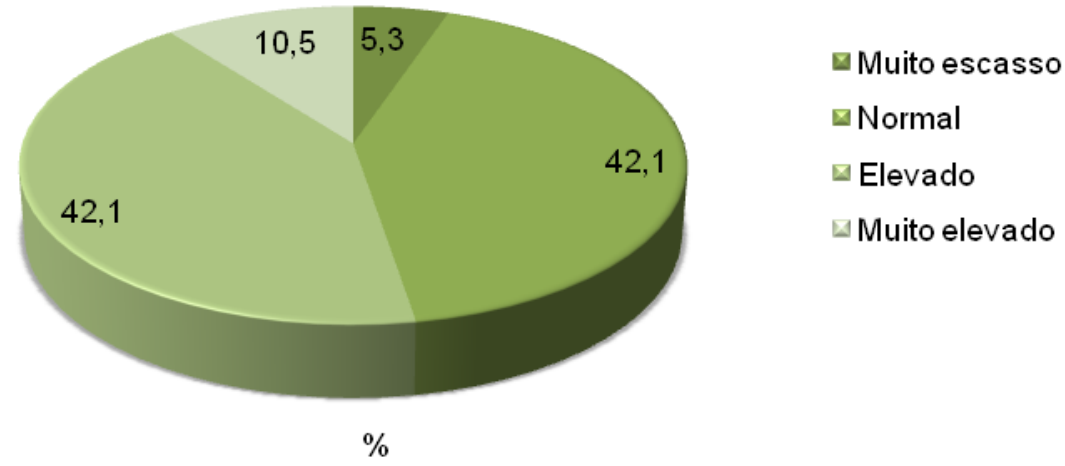

Gráfico 7. Grau de participação dos treinadores de tênis em atividades de formação permanente

Grande parte dos treinadores participa continuamente de atividades de formação permanente, exceto $5,5 \%$ deles, que manifestaram escassa frequência em eventos desta natureza.

Qual a principal dificuldade que você tem para participar de atividades de formação permanente?

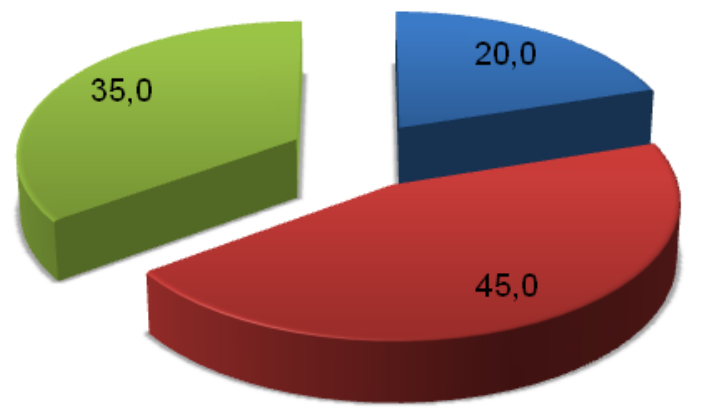

Dificuldades econômicas

Ocupações e responsabilidades familiares

$\square$ Não poder deixar de atender ao seu trabalho

$\%$

Gráfico 8. Fatores dificultadores para a participação dos treinadores de tênis em atividades de formação permanente

Pensar a Prática, Goiânia, v. 17, n. 1, p. 01-294, jan./mar. 2014 
A rotina de trabalho e as ocupações e responsabilidades familiares ocuparam a maior parte da responsabilidade em não permitir ao treinador participar de atividades de formação permanente conforme apreciado. $\mathrm{O}$ tempo extra dispendido em atividades desta natureza parece ser um grande dificultador, visto que afasta o profissional das suas obrigações familiares.

Qual o principal problema na oferta de atividades para melhorar sua formação permanente?

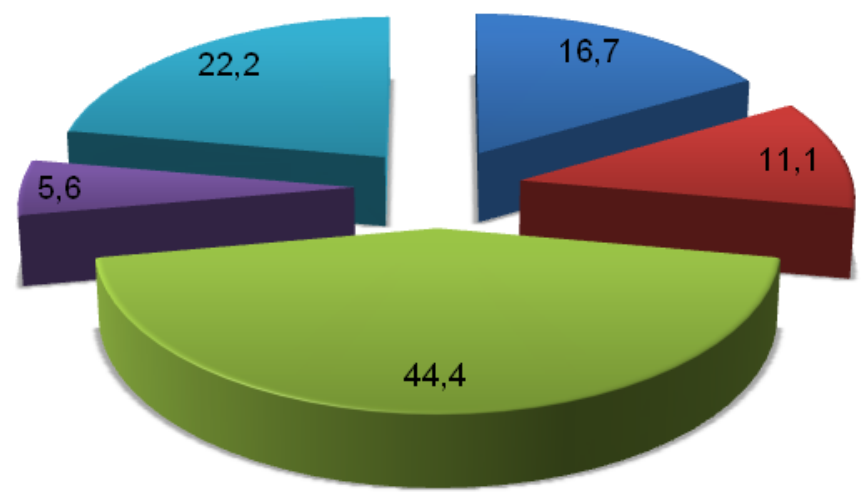

$\%$

घscassa existência de atividades com rigor científico promovidas pelos órgãos federativos

- Escassa adaptação das atividades de índole científica às necessidades e nível de compreensão dos treinadores $\square$ Atividades excessivamente estereotipadas (parecidas, monótonas, repetição de conteú dos)

$\square$ Atividades pouco motivantes

$\square$ Número insuficiente de atividades de aplicação prática

Gráfico 9. Opinião dos treinadores sobre os principais problemas na oferta de atividades de formação permanente

A rotina didática, prática e teórica dos eventos de formação permanente parecem corresponder a grande parte entre os principais problemas na oferta das atividades, com $44,4 \%$ da opinião dos treinadores de tênis. $22,2 \%$ declararam que um número insuficiente de atividades ministradas têm aplicação prática na rotina de trabalho; $16,7 \%$ consideram que são escassas as atividades com rigor científico adotadas nos cursos, e $11,1 \%$ consideram que os conteúdos científicos não são passados para os treinadores de forma clara e objetiva. Ainda entre os principais problemas, 5,6\% declararam que as atividades são pouco motivantes.

Pensar a Prática, Goiânia, v. 17, n. 1, p. 01-294, jan./mar. 2014 


\title{
Conclusões
}

O presente estudo indicou que a faixa etária dos treinadores de tênis pesquisados é a adequada quando comparada aos estudos relacionados à intervenção de treinadores em outros países e de outras modalidades, apresentando em sua bagagem experimental muitos anos de prática de tênis e bom nível de jogo. A expertise adquirida pela prática parece dar suporte de intervenção para a grande maioria dos treinadores, visto que a maior parte deles não tem graduação em Educação física. A renda gerada pela profissão está, em sua maioria, entre 3 e 10 vezes mais que a média da população ocupada do Brasil. O dado é relevante ao passo em que representa uma profissão que não sofre, de maneira geral, o desgaste de uma rotina de vida com vulnerabilidades econômicas, permitindo ao treinador focar-se qualitativamente melhor no seu trabalho. Do ponto de vista formativo, a pesquisa mostra que a maioria dos treinadores de tênis realizou sua formação inicial fora da aprendizagem formal em Ciências do Esporte, fundamentando-se em aprendizagens informais e não-formais para formar-se como treinador, ou ainda optando por aprendizagem formal em outras áreas profissionais como Direito, Odontologia, Administração em Comércio Exterior e Ciências Contábeis, áreas sem muita intersecção com o esporte de rendimento.

Paralelamente ao encontrado em outros estudos, onde profissionais manifestaram que a adequada competência para exercer a função de treinador é aquela em que se encaixa no seu próprio perfil, este estudo evidenciou o mesmo, com os profissionais que possuem graduação em Educação Física salientando a importância da formação para a intervenção como treinador, e com os provisionados percebendo que o nível médio do ensino regular parece ser o adequado para assumir a disciplinas específicas de tênis em encontros de formação permanente.

\section{PROFILE OF BASIC AND PERMANENT COACHING OF HIGH PER- FORMANCE TENNIS COACHES IN BRAZIL}

\begin{abstract}
The aim of this study was to describe the initial and permanent coaching of high performance tennis coaches in Brazil. Had a sample of 24 male coaches, aged $38.58 \pm 8.95$ years. It was observed that the age of tennis coaches is adequate, with many years of practice and good level of tennis play. The expertise gained by the practice seems to support intervention for the vast majority of coaches. The tennis coaches are based on informal learning and non-formal; they participate in continuing education activities, but identify constraints to participation and notice other organizational failures.
\end{abstract}

Key words: Human resources formation, tennis, athletic performance.

Pensar a Prática, Goiânia, v. 17, n. 1, p. 01-294, jan./mar. 2014 13 


\section{PERFIL DE FORMACIÓN INICIAL Y PERMANENTE DEL ENTRENA- DOR DE TÊNIS DE BRAZIL}

\section{Resumen}

El objetivo de este estudio fue describir la formación inicial y continua de los entrenadores de tenis de alto rendimiento en Brasil. Una muestra de 24 entrenadores de los varones, con edades 38,58 $\pm 8,95$ años. Se observó que los entrenadores de tenis grupo de edad es adecuada, con muchos años de práctica y el buen nivel de juego de tenis. La experiencia adquirida por la práctica parece apoyar la intervención para la gran mayoría de los entrenadores. Los entrenadores de tenis se basa en el aprendizaje informal y no formal, participar en actividades de formación permanente, pero identifican las limitaciones a la participación, otro aviso de fallos de organización.

Palabras clave: Formación de recursos humanos, tênis, rendimiento atlético.

\section{Referências}

ATP, Association of Tennis Players. Disponível em: http://www.atpworldtour.com. Londres, Inglaterra. Acessado em: 30 de abril de 2010.

BALBINOTTI, C. A. A. A formação técnica do jogador de tênis: um estudo sobre jovens tenistas brasileiros. Porto: Universidade do Porto, 2003. 133p. Tese (Doutorado) - Programa de Doutorado em Ciências do Desporto, Faculdade de Ciências do Desporto e Educação Física, Universidade do Porto, Porto, 2003.

BALBINOTTI, Marcos Adelar Abaide; BALBINOTTI, Carlos Adelar Abaide; MARQUES, António Teixeira; GAYA, Adroaldo Cezar Araújo. Proposição e validação de um instrumento para avaliação do treino técnicodesportivo de jovens tenistas. Revista Brasileira de Educação Física e Esportes. v.18, n.3, p.213-226, 2004a.

BALBINOTTI, Marcos Adelar Abaide; BALBINOTTI, Carlos Adelar Abaide; MARQUES, António Teixeira; GAYA, Adroaldo Cezar Araújo. O treino técnico desportivo de jovens tenistas brasileiros. Revista Brasileira de Ciências do Esporte. v.26, n.1, p.51-72, 2004 b.

BALBINOTTI, Carlos Adelar Abaide; BALBINOTTI, Marcos Adelar Abaide; MARQUES, António Teixeira; GAYA, Adroaldo Cezar Araújo. O treino técnico desportivo do tenista infanto-juvenil (13-16 anos): um estudo 
descritivo-exploratório com três grupos submetidos a diferentes cargas horárias de treino semanais. Revista Brasileira de Ciências do Esporte. v.29, n.2, p.185-201, 2008.

BELLUCCI sem técnico. Diário Catarinense, Florianópolis, 25 outubro. 2010. Esportes, p. 8.

BERLINER, D. In pursuit of the expert pedagogue. Educational Research, v.15, n.7, p.5-13, 1986.

CASSIDY, T., JONES, R., POTRAC, P. Understanding sports coaching: the social, cultural and pedagogical foundations of coaching practice. $2^{\mathrm{a}}$ ed. Londres: Routledge, 2009.

CBT. Confederação Brasileira de Tênis. Disponível em: http://www.cbt.esp.br/. São Paulo, Brasil. Acessado em: 30 de abril de 2010.

CERVO, A. L.; BERVIAN P. A. Metodologia científica. $3^{\text {a }}$ ed. São Paulo: McGraw-Hill, 1983. 249p.

CÔTÉ, J. The Development of Coaching Knowledge. International Journal of Sports Science \& Coaching, v.1, n.3, p. 217-222, 2006.

CUSHION, C. J.; JONES, R.L. A systematic observation of professional top-level youth soccer coaches. Journal of sport behavior, v. 24, n. 4, p. 354-376, 2001.

CUSHION, C. J.; ARMOUR, K. M.; JONES, R. L. Coach Education and Continuing Professional Development: Experience and Learning to Coach. QUEST, v. 55, n. 3, p. 215-230, 2003.

GOULD, D.; KRANE, V.; GIANNINI, J.; HODGE, K. Educational Needs of Elite US national team, Pan American, and Olympic coaches. Journal of Teaching in Physical Education, v. 9, p. 332-344, 1990.

IBAÑEZ, S.; DELGADO, M.; LORENZO, M.; DEL VILLAR, F.; RIVADENEIRA, M. El entrenador de baloncesto. In: NOGUERA, Delgado; CASAUBÓN, Meina. Experiências de formación de docentes y entrenadores em El âmbito de La actividad física y del deporte. Madrid: Ministerio de educación y Cultura; Consejo Superior de Deportes. 1997. p. 83-129. 
IBGE. Instituto Brasileiro de Geografia e Estatística. Disponível em: http://www.ibge.gov.br/. Brasília, Brasil. Acessado em: 16 de fevereiro de 2011.

ISIDRO, A. S. M. Formação de treinadores em Portugal: condições de acesso, valorização da formação inicial e estratégias de formação contínua. Porto: Universidade do Porto, 2009. 106p. Dissertação (Mestrado) Faculdade de Desporto, $2^{\circ}$ Ciclo em Desporto para Crianças e Jovens, Universidade do Porto, Porto, 2009.

JONES, R. L. Sports coaching as educator: Re-conceptualising sports coaching. London: Routledge, 2006.

KIST, César. Tênis Show. Porto Alegre: 2007. Entrevista concedida à Cláudia Coutinho. Disponível em: <http://tenisshow.clicrbs.com.br/entrevistas_det.php?id=43>. Acessado em: 15 de fevereiro de 2011.

KIRK, D.; GORELY, T. Challenging thinking about the relationship between school physical education and sport performance. European Physical Education Review, v.6 (2), p.119-134, 2000.

LEMYRE, F.; TRUDEL, P.; DURAND-BUSH, N. How youth-sport coaches learn to coach. Sport Psychologist, v. 21, p. 191-209, 2007.

LIMA, Marcelo Bittencourt Neiva de. Formação do treinador de tênis de alto rendimento no Brasil [Dissertação de mestrado]. 242p. Florianópolis: Universidade do Estado de Santa Catarina, Mestrado em Ciências do Movimento Humano; 2011.

MCCALLISTER, S. G.; BLIND, E. M.; KOLENBRANDER, B. Problematic aspects of the role of youth sport coach. International Sport Journal, v. 4, p. 9-26, 2000.

NELSON, L. J.; CUSHION, C. J.; POTRAC, P. Formal, non formal and informal coach learning: a holistic conceptualisation. International Journal of Sports Science \& Coaching, v. 1, p. 247-259, 2006.

NORDMANN, L.; SANDNER, H. The diploma-coaches-study at the coaches academy cologne of the german Olympic sport federation. International Journal of Coaching Science, v.3, n.1, p. 69-80, 2009. 
PIÉRON, M. Enseignement des Activités Physiques et Sportives: Observation et Recherche. Lieja: Prensas de la Universidad de Lieja. 1988.

PIÉRON, M. (1993). Analyser l'enseignement pour mieux enseigner (Dossiers EPS, n.16). Paris: Éditions Revue EPS, 1993.

RAMOS, L. A. La evolución el pensamiento docente de los profesores de Educación Física a través de un programa de supervisión orientado a la reflexión en la acción y sobre la acción. Extremadura: Universidad de Extremadura, 1999. Tese (Doutorado) - Facultad de Ciencias Del Deporte, Departamento de Didáctica de la Expresión Musical, Plástica y Corporal, Universidad de Extremadura, Extremadura, 1999.

RESENDE, R. Análise dos processos formativos do treinador desportivo: um estudo multidimensional aplicado a treinadores de voleibol. A Coruña: Universidade da Coruña, 2009. Tese (Doutorado) Faculdad de Ciencias do Deporte e a Educación Fisica, Universidade da Coruña, A Coruña, 2009.

SFARD, A. On two metaphors for learning and the dangers of choosing just one. Educational Researcher, v. 27, n. 2, p. 4-13, 1998.

SIEDENTOP, D., ELDAR, E. Expertise, experience and effectiveness. Journal of Teaching in Physical Education, n. 8, p.254-260, 1989.

THOMAS, J. R; NELSON, J. K. Métodos de pesquisa em atividade física. 3 ed. Porto Alegre: Artmed, 2002.

TRUDEL, P.; GILBERT, W. Coaching and Coach Education. In: Kirk, D.; Macdonald, D.; O'Sullivan, M. M. (Eds.). The Handbook of Physical Education. London: Sage, 2006. p. 516-539.

VARGAS-TONSING, T. M. Coaches: preferences for continuing coaching education. International Journal of Sports Sciences \& Coaching, v. 2, n. 1, p. 25-35, 2007.

WTA TOUR, Women's Tennis Association. Disponível em: <http://www.sonyericssonwtatour.com>. Saint Petersburg, Estados Unidos. Acessado em: 31 de maio de 2010. 
WOODMAN, L. Coaching: Science, an art, an emerging profession. Sport Science Review, v. 2, n. 2, p. 1-13, 1993.

WRIGHT, T.; TRUDEL, P.; CULVER, D. Learning How to coach: the different learning situations reported by youth ice hockey coaches. Physical Education and Sport Pedagogy, v. 12, n. 3, p.127-144, 2007.

Recebido em: 22/02/2011

Revisado em: 25/08/2012

Aprovado em: 18/01/2013

Endereço para correspondência:

marceloneivadelima@ hotmail.com

Marcelo Bittencourt Neiva de Lima

Universidade do Estado de Santa Catarina - UDESC

Av. Madre Benvenuta, 2007

Itacorubi - Florianópolis - SC

Pensar a Prática, Goiânia, v. 17, n. 1, p. 01-294, jan./mar. 2014 